\title{
EFECTOS DEL RALEO EN LA ESTRUCTURA DEL RODAL, UNA CONTRI- BUCION AL MANEJO DE LAS PLANTACIONES DE PINO INSIGNE, PINUS RADIATA D.DON.
}

\author{
R. Peters* \\ C.D. Oxf.: (568)
}

\section{R E S U M E N}

El volumen de madera a extraerse durante un raleo, las dimensiones del producto y los cambios estructurales que experimenta un rodal con dicha intervención, son elementos importantes en el proceso de decidir técnica y económicamente la mejor oportunidad de una intervención de manejo.

En este trabajo se desarrollaron aquellas funciones que, a partir de los parámetros que caracterizan una plantación de pino insigne y según el grado de intensidad del raleo, permiten estimar los parámetros, tanto del producto del raleo como del rodal que queda en pie.

\section{S U M M A R Y}

Product dimensions and timber volume removed during a thinning operation together with the subsequent change in stand structure are important aspects to be considered when deciding upon the techniques and economics of intermediate cuts.

Accordingly, mathematical functions, useful to thinning operations were developed from studies based on Monterrey pine stands. In practice, once the actual stand parameters of a pole size Monterrey pine stand are known, and the thinning intensity established, these functions can be used to estimate the size of material to be removed as well as the characteristics of the residual stand.

\section{ZUS A M M E NF A S S UNG}

Die bei einer Durchforstung zu erntende Holzmasse, die Dimensionen der Entnahme und die strukturellen Veränderungen als Folge dieses

(*) Dr. Ingeniero Forestal, Profesor de Mensura Forestal de la Universidad Austral de Chile, Decano de Facultad de Ingeniería Forestal. Casilla 567, Valdivia-Chile.
Eingriffes sind wichtige Beurteilungen des technischen und wirtschaftlichen Entscheidungsprozesses des besten Durchforstungszeitpunktes.

In der vorliegenden Abhandlung wurden jene Funktionen entwickelt, welche auf Grund der in einem Pinus radiata-Bestandes existierenden Grössen und des Durchforstungsgrades die Schätzung der Dimensionen sowohl der Entnahme als auch die des verbleibenden Bestandes ermöglicht.

\section{INTRODUCCION}

El conocimiento de los cambios estructurales que experimenta un rodal con una intervención de raleo reviste fundamental importancia para el manejo del bosque. En la decisión de ralear, además de factores de tipo técnico, concurren factores de costos e ingresos que para poder ser considerados, requieren de una estimación más o menos exacta de los rendimientos y características físicas del producto raleado.

Asimismo, la evaluación de los cambios estructurales que sufre un rodal con una intervención de raleo, es una etapa importante en el desarrollo de estudios de crecimiento.

El objetivo de este trabajo es justamente proporcionar al forestal los elementos de juicio necesarios que le permitan, conociendo el estado actual del rodal, estimar los parámetros que caracterizan tanto la porción de rodal que se explota como la que queda en pie, de acuerdo al grado de intensidad de la intervención.

\section{MATERIAL Y METODO}

El trabajo se desarrolló sobre la base de 60 parcelas de rendimiento y de raleo, establecidas dentro del proyecto "Factores y coeficientes para pino insigne", estudio realizado por el Instituto Forestal entre los años 1969 y 1972. Cada parcela, cuya superficie fluctuó entre 0.1 y 0.25 has, se midió en pie antes de ser raleada. 
se raleó y se midieron nuevamente tanto el rodal que quedó en pie como el producto del raleo.

Los datos originales cubren un rango diametral de 12 a $30 \mathrm{~cm}$ y un rango de intensidades de raleo que va desde el $7 \%$ hasta el $43 \%$ en área basal. Todos los valores que excedan este límite son producto de extrapolación.

A través de regresiones apropiadas, basadas en cálculos similares de GRUT (1970), se desarrollaron varias funciones que reflejan los cambios de estructura de un rodal con estas intervenciones.

Se distinguen como consecuencia de la intervención de raleo dos estados de rodal. Una situación original antes del raleo, cuyos parámetros se identifican por un número uno (D1, H1, $\mathrm{N} 1$, G1, V1) y un estado posterior al raleo, caracterizado en su simbología por un número dos (D2 H2, N2, G2, V 2). Finalmente, los parámetros de los árboles explotados durante el raleo se presentan con un número tres junto al correspondiente símbolo (D3, H3, N3, G3, V3).

\section{R E S U L T A D O S}

\subsection{Efecto del raleo sobre el diámetro medio del rodal.}

En Chile el raleo más generalizado en las plantaciones de pino insigne es el tipo por lo bajo. Esto significa que el diámetro de los árboles explotados del bosque es menor que el diámetro original medio del rodal. El rodal que permanece tiene en consecuencia, un diámetro mayor que el original del rodal.

De acuerdo a los resultados de CR A IB (1939) y GRUT (1970), la relación entre el diámetro original del rodal y el diámetro medio del rodal que permanece y de los árboles raleados, es de tipo rectilíneo para cada grado de intensidad de raleo.

En este caso se establecieron cuatro ecuaciones para las intensidades de $10,20,30$ y $40 \%$ en área basal. Los correspondientes valores de las constantes $a_{0}$ y $a_{1}$ y sus respectivos coeficientes de correlación, se entregan en el Cuadro 1 .

\section{CUADR O 1}

Relación diámetro medio del rodal que queda ( D 2 ) en función del diámetro medio original del rodal ( D 1 .

Modelo: D2 $=\mathbf{a}_{0}+\mathbf{a}_{1} \mathrm{Dl}$ $\mathrm{a}_{0} \quad \mathrm{a}_{1} \quad \mathrm{r}$

aleo $\%$ de

área basal

Intensidad de

\begin{tabular}{llll}
10 & 0.350628 & 1.068684 & 0.932 \\
20 & 0.825047 & 1.080684 & 0.831 \\
30 & 1.009469 & 1.072682 & 0.874 \\
40 & 2.200658 & & 0.690 \\
\hline
\end{tabular}

De acuerdo a la metodología definida por Grut, los valores de $a_{0}$ y $a_{1}$ se relacionaron con las intensidades de raleo. Se probaron dos tipos de modelos, recta y parábola de $2^{\circ}$ grado, eligiéndose finalmente como las mejores las siguientes expresiones:

$$
\begin{aligned}
\mathrm{a}_{0}=0.40728-0.27814 \mathrm{~T}+0.18826 \mathrm{~T}^{2} \\
\mathrm{r}=0.996
\end{aligned}
$$

donde:

$\mathrm{T}=$ grado de intensidad de raleo en porcentaje de área basal dividido por 10 .

Reemplazando las expresiones para $a_{0}$ y $a_{1}$ en el modelo original (1), se llega finalmente a aquella ecuación que entrega directamente el diámetro medio del rodal que permanece en pie en función de la intensidad de raleo y del diámetro medio original del rodal intervenido. La expresión resultantes es:

$$
\begin{aligned}
& \mathrm{D} 2=0.40728-0.27814 \mathrm{~T}+0.18826 \mathrm{~T}_{-}^{2}+ \\
& +0.00880 \mathrm{~T} \mathrm{D} 1+1.06058 \\
& \mathrm{D} 1
\end{aligned}
$$

Tanto en el Cuadro 2 como en el Gráfico 1 está representada esta ecuación para facilitar su aplicación. 


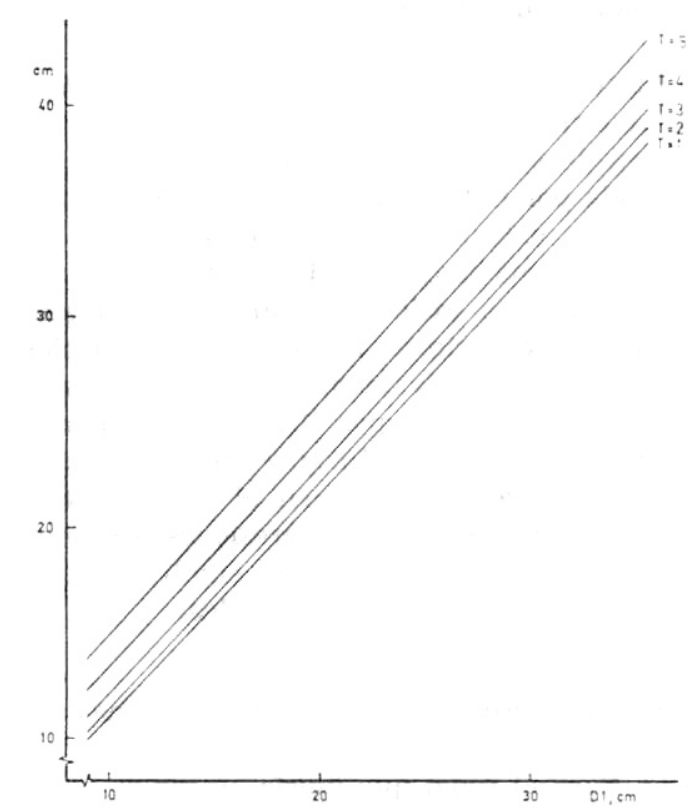

Gráfico 1.- Diámetro medio del rodal raleado (D2) en función del diámetro del rodal antes del raleo (D1) y de la intensidad de raleo ( $T$ ).

\subsection{Efecto del raleo sobre la altura media del rodal}

Otro aspecto de interés es definir el efecto que el raleo tiene sobre la altura media del rodal. La variación de altura media se expresó en función del cambio experimentado por el diámetro medio del rodal. Esta relación se ajusta a una línea recta obteniéndose la siguiente expresión:

$$
\mathrm{dH}=0.25591+0.17839 \mathrm{dD} \quad \mathrm{r}=0.863
$$

donde:

$$
\begin{aligned}
\mathrm{dH}= & \text { incremento en altura después del ra- } \\
& \text { leo, } \mathrm{m} . \\
\mathrm{dD}= & \text { variación experimentada por el diáme- } \\
& \text { metro medio con el raleo, cm. }
\end{aligned}
$$

Como:

\begin{tabular}{|c|c|c|c|c|c|}
\hline \multirow{3}{*}{$\begin{array}{l}\text { Diámetro } \\
\text { medio, cm } \\
\text { (D1) }\end{array}$} & \multicolumn{5}{|c|}{ Intensidad de raleo en porcentaje de área basal } \\
\hline & 10 & 20 & 30 & 40 & 50 \\
\hline & \multicolumn{5}{|c|}{ Diámetro medio del rodal después del raleo, cm (D2) } \\
\hline 10 & 11.0 & 11.4 & 12.1 & 13.3 & 14.8 \\
\hline 11 & 12.1 & 12.5 & 13.2 & 14.4 & 15.9 \\
\hline 12 & 13.1 & 13.5 & 14.3 & 15.4 & 17.0 \\
\hline 13 & 14.2 & 14.6 & 15.4 & 16.5 & 18.1 \\
\hline 14 & 15.3 & 15.7 & 16.5 & 17.6 & 19.2 \\
\hline 15 & 16.4 & 16.8 & 17.6 & 18.7 & 20.3 \\
\hline 16 & 17.4 & 17.8 & 18.7 & 19.8 & 21.4 \\
\hline 17 & 18.5 & 18.9 & 19.7 & 20.9 & 22.5 \\
\hline 18 & 19.6 & 20.0 & 20.8 & 22.0 & 23.6 \\
\hline 19 & 20.6 & 21.1 & 21.9 & 23.1 & 24.7 \\
\hline 20 & 21.7 & 22.2 & 23.0 & 24.2 & 25.8 \\
\hline 21 & 22.8 & 23.2 & 24.1 & 25.3 & 26.9 \\
\hline 22 & 23.8 & 24.3 & 25.2 & 26.4 & 28.0 \\
\hline 23 & 24.9 & 25.4 & 26.3 & 27.5 & 29.1 \\
\hline 24 & 26.0 & 26.5 & 27.4 & 28.6 & 30.2 \\
\hline 25 & 27.0 & 27.6 & 28.5 & 29.7 & 31.3 \\
\hline 26 & 28.1 & 28.6 & 29.6 & 30.8 & 32.4 \\
\hline 27 & 29.2 & 29.7 & 30.6 & 31.9 & 33.5 \\
\hline 28 & 30.3 & 30.8 & 31.7 & 33.0 & 34.6 \\
\hline 29 & 31.3 & 31.9 & 32.8 & 34.1 & 35.8 \\
\hline 30 & 32.4 & 32.9 & 33.9 & 35.2 & 36.9 \\
\hline 31 & 33.5 & 34.0 & 35.0 & 36.3 & 38.0 \\
\hline 32 & 34.5 & 35.1 & 36.0 & 37.4 & 39.1 \\
\hline 33 & 35.6 & 36.2 & 37.1 & 38.5 & 40.2 \\
\hline 34 & 36.7 & 37.3 & 38.2 & 39.6 & 41.3 \\
\hline 35 & 37.7 & 38.3 & 39.3 & 40.6 & 42.4 \\
\hline
\end{tabular}

$\mathrm{dH}=\mathrm{H} 2-\mathrm{H} 1$ y $\mathrm{dD}=\mathrm{D} 2-\mathrm{D} 1$

\section{CUADRO 2.}

Diámetro medio del rodal raleado (D2) en función de la intensidad del raleo y del diámetro medio del rodal antes de la intervención (D1). 
donde:

H1 = altura media antes del raleo

H2 = altura media después del raleo

La expresión (5) puede transformarse en:

$\mathrm{H} 2=0.2559-+0.17839 \mathrm{dD}-\mathrm{H} 1$

Por motivos prácticos se incluye una tabla que indica la variación en altura media que experimenta un rodal por cada centímetro de variacinó del diámetro medio, Cuadro 3 y su correspondiente representación gráfica. Gráfico 2 .

\section{CUADRO 3}

Variación de la altura media de un rodal (dH) de acuerdo al aumento del diámetro medio (dD).

\begin{tabular}{cc}
\hline $\begin{array}{c}\text { Aumento en diámetro } \\
\mathrm{cm}\end{array}$ & $\begin{array}{c}\text { Aumento en altura } \\
\mathrm{m}\end{array}$ \\
\hline $\mathrm{dH}$ & $\mathrm{dD}$ \\
$0.1-0.5$ & 0.3 \\
$0.6-1.2$ & 0.4 \\
$1.3-1.6$ & 0.5 \\
$1.7-2.1$ & 0.6 \\
$2.2-2.7$ & 0.7 \\
$2.8-3.3$ & 0.8 \\
$3.4-3.8$ & 0.9 \\
$3.9-4.4$ & 1.0 \\
$4.5-5.0$ & 1.1 \\
$5.2-5.5$ & 1.2 \\
$5.6-6.0$ & 1.3 \\
$6.1-6.5$ & 1.4 \\
$6.6-7.0$ & 1.5 \\
\hline
\end{tabular}

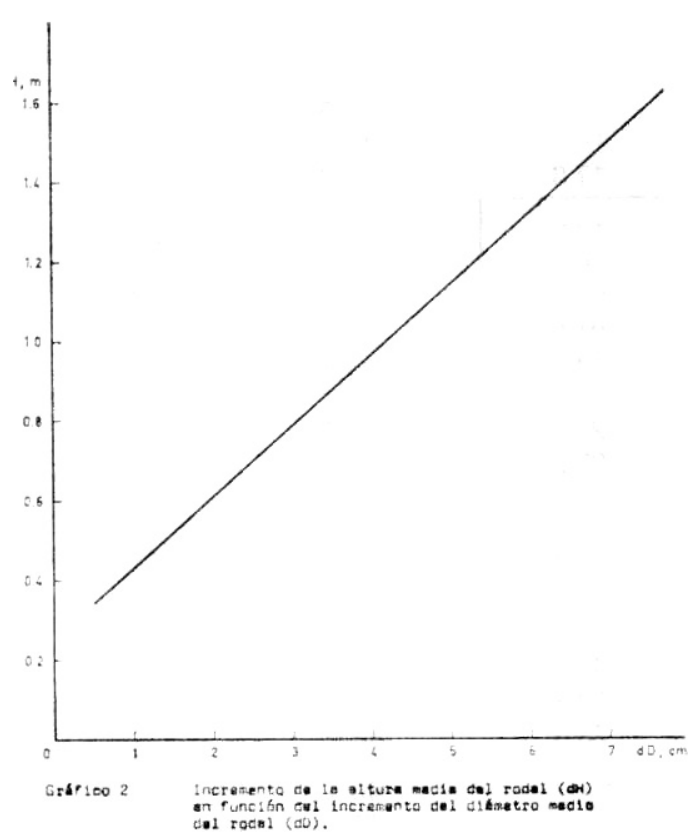

\subsection{Volumen del raleo.}

El volumen por há de un rodal se puede calcular directamente a partir de funciones de volumen de rodal. Siguiendo la metodología desarrollada por GRUT (1970) se comprobó que era necesario calcular dos funciones de rodal por separado, una para el rodal antes de ser raleado y otra para estimar la cantidad de madera, producto del primer raleo. Ambas ecuaciones difieren significativamente entre sí, confirmándose lo determinado por GRUT (1970) para las plantaciones de pino insigne en Sudáfrica. La prueba aplicada se basó en el test de Barlett que establece que dos regresiones se pueden agrupar si no hay diferencias significativas entre sus valores medios residuales.

Se obtuvieron las siguientes ecuaciones:

\subsubsection{Para el rodal que queda en pie}

$$
\mathrm{V} 2=53.44+0.2688 \mathrm{G} 2 \quad \mathrm{x} \quad \mathrm{H} 1
$$

donde:

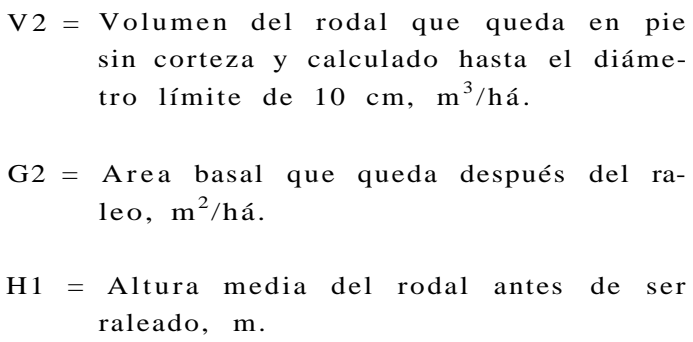
sin corteza y calculado hasta el diámetro límite de $10 \mathrm{~cm}, \mathrm{~m}^{3} / \mathrm{há}$.

G2 = Area basal que queda después del raleo, $\mathrm{m}^{2} / \mathrm{há}$.

H1 = Altura media del rodal antes de ser raleado, $\mathrm{m}$.

Coeficiente de correlación: 0.977

Error estándar de la estimación: 23.0 metros cúbicos por hectárea, $8.7 \%$.

\subsubsection{Para el producto del raleo. \\ $\mathrm{V} 3=-1.19+0.3020 \mathrm{G} 3 \mathrm{H} 1$ \\ donde:}

$\mathrm{V} 3=$ Volumen explotado en el primer raleo, $\mathrm{m}^{3} /$ há .

G3 = Area basal extraída en el primer raleo, $\mathrm{m}^{2} /$ há.

Coeficiente de correlación: 0.886

Error estándar de la estimación: 14.3 metros cúbicos por há, $13.2 \%$. 


\section{APLICACION DE LAS FUNCIONES}

Si se desea determinar el rendimiento de un raleo y los cambios de estructura que sufrirá el rodal, es necesario disponer de los siguientes antecedentes:

a) Diámetro y altura media del rodal.

b) Número de árboles o área basal del rodal.

c) Intensidad del raleo en porcentaje de área basal.

$$
\mathrm{G}=\mathrm{N} \frac{\pi \mathrm{D}^{2}}{4}=1580 \frac{3.1416(0.16)^{2}}{4}=31.8 \mathrm{~m}^{2} / \mathrm{há}
$$
neral:
$\mathrm{N}=\mathrm{Número}$ de árboles por há. Los valores tabulados correspondientes a esta expresión se incluyen en el Cuadro 4.

En este caso el área basal del rodal sin intervenir (G1) alcanza a 31.8 metros cuadrados por há. Un raleo del $20 \%$ significa extraer $6.4 \mathrm{~m}^{2}$ por há (G3).

4.1 Parámetros de la porción del rodal que permanece.

4.1.1 Volumen. El volumen que queda en pie se obtiene aplicando la ecuación (7)

donde:

$$
\begin{aligned}
\mathrm{G} 2= & \mathrm{G} 1-03=31.8-6.4=25.4 \\
\mathrm{~V} 2= & 53.44+0.2688 \times 25.4 \times 22= \\
= & 203.6 \mathrm{~m}^{3} / \mathrm{há} .
\end{aligned}
$$

4.1.2 Diámetro medio. El diámetro medio del rodal que queda en pie se obtiene aplicando la ecuación (4) o más simplemente, leyendo el valor correspondiente en el Cuadro 2. En este caso para un diámetro inicial (D1) de $16 \mathrm{~cm}$ y una intensidad de raleo del $20 \%$, el diámetro del rodal (D2) es $17.8 \mathrm{~cm}$.

4.1.3 Altura media. La altura media del rodal se deriva de la ecuación (6) o bien aplicando el Cuadro 3. Allí se establece que para un incremento en diámetro medio de $1.8 \mathrm{~cm}$ la altura media sufre un incremento de $0.6 \mathrm{~m}$, por 10 tanto, la nueva altura media (H2) es de $22.6 \mathrm{~m}$.

4.1.4 Número de árboles por hectárea. El número de árboles que queda en pie (N2) es de
El siguiente ejemplo ilustra como se usan las ecuaciones desarrolladas. Se asume que un rodal con 1580 árboles por hectárea (N 1), un diámetro medio (D1) de $16 \mathrm{~cm}$ y una altura media (H1) de 22 metros va a ser raleado con una intensidad del 20\%. ¿Cuántos árboles deben extraerse, qué volumen se obtiene del raleo y cómo cambia la estructura del rodal que queda?

En primer lugar se debe calcular el área basal por hectárea a través de la expresión ge-

estimación exacta a través de la ecuación (9), donde se despeja el valor de N. También es posible determinarlo en forma aproximada en el Cuadro 4.

j

$$
\mathrm{N} 2=\frac{25.4 \times 4}{3.1416 \times(0.178)^{2}}=1021
$$

En este caso, para $25.4 \mathrm{~m}^{2} / \mathrm{há}$ de área basal y $17.8 \mathrm{~cm}$ de diámetro medio, el número de árboles por hectárea (N2) es 1021.

\subsection{Parámetros de los árboles explotados.}

4.2.1 Volumen. El volumen producto del raleo (V3) se calcula con la expresión (8).

$\mathrm{V} 3=-1.19+, 0.3020 \times 6.4 \times 22=41.3 \mathrm{~m}^{3} / \mathrm{há}$,

4.2.2 Número de árboles por hectárea. Una de las mayores dificultades que debe enfrentar un técnico forestal al realizar el marqueo de un raleo es estimar el número de árboles que representa el grado de intensidad en área basal elegido. A través de la metodología expuesta se facilitará enormemente. El número de árboles por há antes del raleo son 1580, después de la intervención quedan según nuestra estimación 1021, por lo tanto para alcanzar el $20 \%$ del área basal deben sacarse 559 árboles por hectárea,

$$
\begin{aligned}
& \mathrm{N} 3=\mathrm{N} 1-\mathrm{N} 2 \\
& \mathrm{~N} 3=559 \text { árboles por hectárea. }
\end{aligned}
$$

\subsubsection{Diámetro medio de los árboles raleados.}

Se procede en este caso también usando el Cuadro 4, donde puede obtenerse un valor aproxi- 
C UADRO 4. Area basal por hectárea en función del número de árboles y

el diámetro medio del rodal.

\begin{tabular}{|c|c|c|c|c|c|c|c|c|c|c|c|c|c|c|c|c|c|c|c|c|c|c|c|c|c|}
\hline \multirow{3}{*}{$\begin{array}{c}\text { Diámetro } \\
\text { medio } \\
\mathrm{cm}\end{array}$} & \multicolumn{25}{|c|}{ Número de árboles por hectárea } \\
\hline & 50 & 100 & 150 & 200 & 250 & 300 & 350 & 400 & 450 & 500 & 600 & 700 & 800 & 900 & 1000 & 1100 & 1200 & 1300 & 1400 & 1500 & 1600 & 1700 & 1800 & 1900 & 2000 \\
\hline & \multicolumn{25}{|c|}{ Area basal por hectárea en metros cuadrados } \\
\hline$\ldots 10$ & 0.4 & 0.8 & 1.2 & 1.6 & 2.0 & 2.4 & 2.7 & 3.1 & 3.5 & 3.9 & 4.7 & 5.5 & 6.3 & 7.1 & 7.8 & 8.6 & 9.4 & 10.2 & 11.0 & 11.8 & 12.6 & 13.4 & 14.1 & 14.9 & 15.7 \\
\hline 11 & 0.5 & 1.0 & 1.4 & 1.9 & 2.4 & 2.8 & 3.3 & 3.8 & 4.3 & 4.8 & 5.7 & 6.6 & 7.6 & 8.6 & 9.5 & 10.4 & 11.4 & 12.4 & 13.3 & 14.2 & 15.2 & 16.2 & 17.1 & 18.0 & 19.0 \\
\hline 12 & 0.6 & 1.1 & 1.7 & 2.3 & 2.8 & 3.4 & 4.0 & 4.5 & 5.1 & 5.6 & 6.8 & 7.9 & 9.0 & 10.2 & 11.3 & 12.4 & 13.6 & 14.7 & 15.8 & 17.0 & 18.1 & 19.2 & 20.4 & 21.5 & 22.6 \\
\hline 13 & 0.7 & 1.3 & 2.0 & 2.6 & 3.3 & 4.0 & 4.6 & 5.3 & 6.0 & 6.6 & 8.0 & 9.3 & 10.6 & 11.9 & 13.3 & 14.6 & 15.9 & 17.2 & 18.6 & 19.9 & 21.2 & 22.6 & 23.9 & 25.2 & 26.5 \\
\hline 14 & 0.8 & 1.5 & 2.3 & 3.1 & 3.8 & 4.6 & 5.4 & 6.2 & 6.9 & 7.7 & 9.2 & 10.8 & 12.3 & 13.8 & 15.4 & 16.9 & 18.5 & 20.0 & 21.6 & 23.1 & 24.6 & 26.2 & 27.7 & 29.2 & 30.8 \\
\hline 15 & 0.9 & 1.8 & 2.6 & 3.5 & 4.4 & 5.3 & 6.2 & 7.1 & 8.0 & 8.8 & 10.6 & 12.4 & 14.1 & 15.9 & 17.7 & 19.4 & 21.2 & 23.0 & 24.7 & 26.5 & 28.2 & 30.0 & 31.8 & 33.6 & 35.3 \\
\hline 16 & 1.0 & 2.0 & 3.0 & 4.0 & 5.0 & 6.0 & 7.0 & 8.0 & 9.0 & 10.0 & 12.0 & 14.0 & 16.1 & 18.1 & 20.1 & 22.1 & 24.1 & 26.1 & 28.1 & 30.2 & 32.2 & 34.2 & & & \\
\hline 17 & 1.1 & 2.3 & 3.4 & 4.5 & 5.7 & 6.8 & 7.9 & 9.1 & 10.2 & 11.4 & 13.6 & 15.9 & 18.2 & 20.4 & 22.6 & 25.0 & 27.2 & 29.5 & 31.8 & 34.0 & 36.3 & 38.6 & & & \\
\hline 18 & 1.3 & 2.5 & 3.8 & 5.1 & 6.4 & 7.6 & 8.9 & 10.2 & 11.4 & 12.7 & 15.3 & 17.8 & 20.4 & 22.9 & 25.4 & 28.0 & 30.5 & 33.1 & 35.6 & 38.2 & 40.7 & 43.2 & & & \\
\hline 19 & 1.4 & 2.8 & 4.2 & 5.7 & 7.1 & 8.5 & 9.9 & 11.3 & 12.8 & 14.2 & 17.0 & 19.8 & 22.7 & 25.5 & 28.4 & 31.2 & 34.0 & 36.8 & 39.7 & 42.5 & 45.4 & 48.2 & & & \\
\hline 20 & 1.6 & 3.1 & 4.7 & 6.3 & 7.8 & 9.4 & 11.0 & 12.6 & 14.1 & 15.7 & 18.8 & 22.0 & 25.1 & 28.2 & 31.4 & 34.6 & 37.7 & 40.8 & 44.0 & 47.1 & 50.3 & 53.4 & & & \\
\hline 21 & 1.7 & 3.5 & 5.2 & 6.9 & 8.7 & 10.4 & 12.1 & 13.9 & 15.6 & 17.3 & 20.7 & 24.2 & 27.7 & 31.2 & 34.6 & 38.1 & 41.6 & 45.0 & 48.5 & & & & & & \\
\hline 22 & 1.9 & 3.8 & 5.7 & 7.6 & 9.5 & 11.4 & 13.3 & 15.2 & 17.1 & 19.0 & 22.8 & 26.6 & 30.4 & 34.2 & 38.0 & 41.8 & 45.6 & 49.4 & 53.2 & & & & & & \\
\hline 23 & 2.1 & 4.2 & 6.2 & 8.3 & 10.4 & 12.5 & 14.5 & 16.6 & 18.7 & 20.8 & 24.9 & 29.1 & 33.2 & 37.4 & 41.5 & 45.7 & 49.8 & 54.0 & 58.2 & & & & & & \\
\hline 24 & 2.3 & 4.5 & 6.8 & 9.0 & 11.3 & 13.6 & 15.8 & 18.1 & 20.4 & 22.6 & 27.1 & 31.7 & 36.2 & 40.7 & 45.2 & 49.8 & 54.3 & 58.8 & 63.3 & & & & & & \\
\hline 25 & 2.4 & 4.9 & 7.4 & 9.8 & 12.3 & 14.7 & 17.2 & 19.6 & 22.1 & 24.5 & 29.4 & 34.4 & 39.3 & 44.2 & 49.1 & 54.0 & 58.9 & 63.8 & 68.7 & & & & & & \\
\hline 26 & 2.6 & 5.3 & 8.0 & 10.6 & 13.3 & 15.9 & 18.6 & 21.2 & 23.9 & 26.5 & 31.9 & 37.2 & 42.5 & 47.7 & 53.1 & 58.4 & & & & & & & & & \\
\hline 27 & 2.9 & 5.7 & 8.6 & 11.4 & 14.3 & 17.2 & 20.0 & 22.9 & 25.8 & 28.6 & 34.4 & 40.1 & 45.8 & 51.5 & 57.2 & 63.0 & & & & & & & & & \\
\hline 28 & 3.1 & 6.2 & 9.2 & 12.3 & 15.4 & 18.5 & 21.6 & 24.6 & 27.7 & 30.8 & 36.9 & 43.1 & 49.3 & 55.4 & 61.6 & 67.7 & & & & & & & & & \\
\hline 29 & 3.3 & 6.6 & 9.9 & 13.2 & 16.5 & 19.8 & 23.1 & 26.4 & 29.7 & 33.0 & 39.6 & 46.2 & 52.8 & 59.4 & 66.0 & 72.6 & & & & & & & & & \\
\hline 30 & 3.5 & 7.1 & 10.6 & 14.1 & 17.7 & 21.2 & 24.7 & 28.3 & 31.8 & 35.3 & 42.4 & 49.5 & 56.6 & 63.6 & 70.7 & 77.7 & & & & & & & & & \\
\hline 31 & 3.8 & 7.5 & 11.3 & 15.1 & 18.9 & 22.6 & 26.4 & 30.2 & 34.0 & 37.7 & 45.3 & 52.8 & 60.4 & & & & & & & & & & & & \\
\hline 32 & 4.0 & 8.0 & 12.1 & 16.1 & 20.1 & 24.1 & 28.1 & 32.2 & 36.2 & 40.2 & 48.2 & 56.3 & 64.3 & & & & & & & & & & & & \\
\hline 33 & 4.3 & 8.6 & 12.8 & 17.1 & 21.4 & 25.6 & 29.9 & 34.2 & 38.5 & 42.8 & 51.3 & 59.9 & 68.4 & & & & & & & & & & & & \\
\hline 34 & 4.5 & 9.1 & 13.6 & 18.2 & 22.7 & 27.2 & 31.8 & 36.3 & 40.9 & 45.4 & 54.5 & 63.6 & 72.6 & & & & & & & & & & & & \\
\hline 35 & 4.8 & 9.6 & 14.4 & 19.2 & 24.0 & 28.9 & 33.7 & 38.5 & 43.3 & 48.1 & 57.7 & 67.3 & 77.0 & & & & & & & & & & & & \\
\hline
\end{tabular}


mado. Un cálculo exacto se obtiene despejando el valor D3 de la ecuación (9).

$$
\mathrm{D} 3=\sqrt{\frac{/ \mathrm{G} 3 \times 4}{\mathrm{~N} 3 \times \pi}}=\sqrt{\frac{/ 6.4 \times 4}{559 \times 3.1416} 12.0 \mathrm{~cm}}
$$

El diámetro medio de los árboles extraídos durante el raleo es de 12 centímetros.

\section{$\begin{array}{lllllllllll}R & E & F & E & R & E & N & C & I & A & S\end{array}$}

CRAIB, I.J., 1939: Thinning, pruning and management studies on the main, exotic conifers grown in South Africa. Department of Agricultura and Forestry, Science Bulletin $\mathrm{N}^{\circ} 196$.

GRUT, M., 1970: Pinus radiata, Growth and Economics, A.A. Balkema, Capetown. 